\title{
BMJ Open Prospective association between adherence to the Mediterranean diet and hepatic steatosis: the Swiss CoLaus cohort study
}

\author{
Saman Khalatbari-Soltani (D) , , ${ }^{1,3}$ Pedro Marques-Vidal (D) , ${ }^{3}$ Fumiaki Imamura (D) , ${ }^{4}$ \\ Nita G. Forouhi ${ }^{4}{ }^{4}$
}

To cite: Khalatbari-Soltani S, Marques-Vidal P, Imamura F, et al. Prospective association between adherence to the Mediterranean diet and hepatic steatosis: the Swiss CoLaus cohort study. BMJ Open 2020;10:e040959. doi:10.1136/ bmjopen-2020-040959

- Prepublication history and additional materials for this paper are available online. To view these files, please visit the journal online (http://dx.doi. org/10.1136/bmjopen-2020040959).

FI and NGF contributed equally.

$\mathrm{Fl}$ and NGF are joint senior authors.

Received 27 May 2020

Revised 13 November 2020

Accepted 17 November 2020

Check for updates

(C) Author(s) (or their employer(s)) 2020. Re-use permitted under CC BY. Published by BMJ.

For numbered affiliations see end of article.

Correspondence to Dr Saman Khalatbari-Soltani; saman.khalatbarisoltani@ sydney.edu.au and Professor Nita G. Forouhi; nita.forouhi@mrc-epid.cam. ac.uk

\section{ABSTRACT}

Objective The Mediterranean diet has been promoted as a healthy dietary pattern, but whether the Mediterranean diet may help to prevent hepatic steatosis is not clear.

This study aimed to evaluate the prospective association between adherence to the Mediterranean diet and risk of hepatic steatosis.

Design Population-based prospective cohort study.

Setting The Swiss CoLaus Study.

Participants We evaluated 2288 adults $(65.4 \%$ women, aged $55.8 \pm 10.0$ years) without hepatic steatosis at first follow-up in 2009-2012. Adherence to the Mediterranean diet was scaled as the Mediterranean diet score (MDS) based on the Mediterranean diet pyramid ascertained with responses to Food Frequency Questionnaires.

Outcome measures New onset of hepatic steatosis was ascertained by two indices separately: the Fatty Liver Index (FLI, $\geq 60$ points) and the non-alcoholic fatty liver disease (NAFLD) score ( $\geq-0.640$ points). Prospective associations between adherence to the Mediterranean diet and risk of hepatic steatosis were quantified using Poisson regression. Results During a mean 5.3 years of follow-up, hepatic steatosis was ascertained in $153(6.7 \%)$ participants by FLI criteria and in $208(9.1 \%)$ by NAFLD score. After multivariable adjustment, higher adherence to MDS was associated with lower risk of hepatic steatosis based on FLI: risk ratio $0.84(95 \% \mathrm{Cl} 0.73$ to 0.96$)$ per $1 \mathrm{SD}$ of MDS; 0.85 (0.73 to 0.99) adjusted for BMl; and 0.85 (0.71 to 1.02) adjusted for both BMI and waist circumference. When using NAFLD score, no significant association was found between MDS and risk of hepatic steatosis (0.95 (0.83 to 1.09)).

Conclusion A potential role of the Mediterranean diet in the prevention of hepatic steatosis is suggested by the inverse association observed between adherence to the Mediterranean diet and incidence of hepatic steatosis based on the FLI. The inconsistency of this association when hepatic steatosis was assessed by NAFLD score points to the need for accurate population-level assessment of fatty liver and its physiological markers.

\section{INTRODUCTION}

Hepatic steatosis is the most common cause of liver disease. ${ }^{1}$ In westernised countries, hepatic steatosis affects up to $34 \%$ of the
Strengths and limitations of this study

- This study had the benefit of a relatively large sample size and an average of 5.3 years of follow-up.

- We applied a definition of the Mediterranean diet that has been shown to be valid in a non-Mediterranean population.

- Our ascertainment of hepatic steatosis was based on two indices that have been validated for use in large epidemiological studies.

- We used dietary data measured only once at baseline, and intraindividual variation over time might be present which may weaken the observed associations towards the null; however, dietary intake in CoLaus was relatively stable, suggesting that lack of repeated dietary measures is unlikely to alter our findings substantially.

- The precision of the two hepatic steatosis indices used is different and may be influenced by the presence of steatohepatitis or advanced liver fibrosis.

general population and up to $74 \%$ of obese individuals, depending on the definition used. $^{2-4}$ Hepatic steatosis-fat content of more than $5 \%$ of liver volume-is the first recognisable stage of non-alcoholic fatty liver disease (NAFLD). ${ }^{1}$ Hepatic steatosis, particularly NAFLD, may progress to end-stage liver disease including fibrosis, cirrhosis and hepatocellular carcinoma. ${ }^{5}$ Moreover, as hepatic steatosis increases the risk of metabolic syndrome, type 2 diabetes and cardiovascular disease (CVD), its prevention is of public health importance. ${ }^{6}$ An unhealthy dietary pattern remains one of the primary targets of lifestyle modification for the prevention and management of hepatic steatosis and NAFLD. 78

The Mediterranean diet has been recently recommended for treatment of NAFLD. ${ }^{9}$ In recent years, a growing body of evidence supports the idea that the Mediterranean diet may be the reference nutritional profile 
for the prevention of hepatic steatosis development. ${ }^{10-12}$ Adherence to the Mediterranean diet has been reported to have a beneficial impact on risks of CVD,${ }^{13} 14$ type 2 diabetes ${ }^{15}$ and metabolic syndrome. ${ }^{16}$ Trial evidence demonstrated the potential benefits of the Mediterranean diet against progress of hepatic steatosis focusing on individuals with existing hepatic steatosis, either alone $^{17-22}$ or associated with metabolic risk factors such as obesity or diabetes. ${ }^{22-25}$ Research among those without clinically manifest hepatic steatosis is restricted to observational evidence, reporting an inverse association that greater adherence to a Mediterranean diet is associated with lower prevalence of hepatic steatosis. ${ }^{26}{ }^{27}$ However, the cross-sectional design of these studies limits inference for causal associations and can be used mainly for hypothesis generation. Relevant longitudinal evidence for the primary prevention of hepatic steatosis or NAFLD has been reported only by the Framingham Heart Study, with a significant inverse association of adherence to the Mediterranean diet with risk of hepatic steatosis in 1521 adults over 6 years of follow-up, ${ }^{28}$ but evidence is lacking in Europe.

Given the limited evidence from population-based epidemiological studies thus far, we aimed to investigate the prospective association between adherence to the Mediterranean diet and the risk of developing hepatic steatosis among adults without clinically manifest hepatic steatosis. We hypothesised that greater adherence to the Mediterranean diet would reduce the risk of hepatic steatosis.

\section{METHODS}

\section{Study population}

We evaluated participants in the CoLaus Study, an ongoing population-based cohort investigating the clinical, biological and genetic determinants of CVD in the city of Lausanne, Switzerland. ${ }^{29}$ Inclusion criteria of the recruitment were adults of European origin, aged 35-75 years. ${ }^{29}$ There were three study phases: baseline recruitment in 2003-2006 ( $\mathrm{n}=6733$ ), the first follow-up in 2009$2012(\mathrm{n}=5064)$ and the second follow-up in 2014-2017 $(n=4881)$. We conducted dietary assessment at the first follow-up and therefore considered the first follow-up as the study baseline. Fatty Liver Index (FLI) and NAFLD score, two indices of hepatic steatosis, were available at baseline. ${ }^{27}$ If participants met the joint criterion of FLI $\geq 60$ or NAFLD score $\geq-0.640$ at baseline, we excluded them as prevalent cases (see below) $(n=2036)$. We also excluded participants with missing information on diet, outcome and covariates $(\mathrm{n}=740)$ (online supplemental figure S1).

\section{Patient and public involvement}

Patients and/or the public were not involved in the design, conduct, reporting or dissemination plans of this research.

\section{Dietary assessment}

Participants completed a self-administered, 97-item, semiquantitative Food Frequency Questionnaire (FFQ) about their habitual dietary intake over the last 4 weeks, ${ }^{30}$ the validity of which had been assessed in canton Geneva against 24-hour recalls. ${ }^{30} 31$ For each item, participants were instructed to report consumption frequencies by selecting one of the seven frequency options from 'less than once during the last 4 weeks' to 'two or more times per day' and by selecting a usual serving size (smaller, equal or bigger to a reference size).

\section{Mediterranean diet scores}

We derived the pyramid-based Mediterranean diet score (MDS) as a measure of adherence to the Mediterranean diet from responses to the FFQ as we conducted previously. ${ }^{27}$ This MDS is based on the Mediterranean dietary pyramid proposed by the Mediterranean Diet Foundation for both Mediterranean and non-Mediterranean countries, and accounting for the traditional Mediterranean diet, contemporary lifestyle and food environment. ${ }^{32} \mathrm{We}$ have previously reported that this MDS scoring algorithm predicted CVD incidence, ${ }^{33}$ as well as the prevalence of hepatic steatosis ${ }^{27}$ in non-Mediterranean populations. Briefly, a continuous score of $0-1$ was assigned for each recommended level of the 15 components of the pyramid (vegetables, legumes and fish as healthy items; red meat, processed meat, potato and sweets as unhealthy items; and fruits, nuts, cereals, eggs, dairy, white meat and alcoholic beverages as items for which moderate consumption was recommended). The resulting MDS ranges between 0 and 15 on a continuous scale. The MDS calculation was adjusted to an energy intake of $2000 \mathrm{kcal} / \mathrm{d}$ (8.37 $\mathrm{MJ} /$ day) by applying a regression-residual technique for energy adjustment to each food group variable. ${ }^{33} 34$

\section{Ascertainment of hepatic steatosis}

Two indices of hepatic steatosis were evaluated: $\mathrm{FLI}^{35}$ and NAFLD liver fat score. ${ }^{36}$ FLI was calculated based on a logistic function including body mass index (BMI), waist circumference, fasting triglycerides and gamma-glutamyl transferase (GGT) levels as follows:

$\mathrm{FLI}=1 /\left(1+\mathrm{e}^{-(0.953 \times \ln }\right.$ (triglycerides) $+0.139 \times \mathrm{BMI}+0.718 \times \mathrm{ln}$ $($ GGT $)+0.053 \times$ waist circumference-15.745) $)$.

FLI $\times 100$ ranges from 0 to 100 . Presence of hepatic steatosis was defined by FLI $\geq 60$, a value with a sensitivity of $61 \%$ and a specificity of $86 \%{ }^{35}$ FLI was tested previously in comparison to ultrasonography with an area under the receiver operating characteristic curve of 0.78 (OR 95\% CI: 0.77 to 0.83 ). ${ }^{37}$

The NAFLD score was calculated based on an algorithm including a logistic function with the presence of metabolic syndrome defined by criteria of International Diabetes Federation, ${ }^{38}$ presence of type 2 diabetes, and fasting concentrations of insulin, aspartate aminotransferase (AST) and the AST/alanine transaminase (ALT) ratio: 
NAFLD score $=-2.89+1.18 \times$ metabolic syndrome (yes / no) $+0.45 \times$ type 2 diabetes (yes $/$ no) $+0.15 \times$ fasting insulin $(\mathrm{mU} / \mathrm{L})+0.04 \times$ fasting AST $(\mathrm{U} / \mathrm{L})-0.94 \times$ AST $/$ ALT .

Presence of hepatic steatosis was defined by a NAFLD score $\geq-0.640$, a value with a sensitivity of $86 \%$ and a specificity of $71 \%$, when compared with proton MRI. ${ }^{36}$

\section{Assessment of covariates at baseline}

Sociodemographic, lifestyle and health characteristics were collected by self-administered questionnaires. Age, sex, marital status, occupational status and educational level were included as indicators of sociodemographic condition. Smoking status was classified as 'never', 'former' and 'current'. Alcohol consumption was assessed by the number of alcoholic beverage units consumed in the past week and further categorised as 'abstainers' $(0$ unit/week), 'moderate' (1-21 units/week for men, 1-14 for women) and 'heavy' (>21 units/week for men, $>14$ for women) drinkers (1 unit corresponds to $8 \mathrm{~g}$ of alcohol). Physical activity was assessed with a self-administered quantitative physical activity frequency questionnaire. ${ }^{39}$ Health characteristics included presence of metabolic syndrome and family history of diabetes. Anthropometric and blood pressure measurements were obtained using standard procedures and equipment as previously described. ${ }^{29}$ Plasma triglycerides, high-density lipoprotein cholesterol and glucose were measured using standard enzymatic methods, and ALT, AST and GGT were measured using reference methods as standardised by the International Federation of Clinical Chemistry.

\section{Statistical analysis}

Statistical analyses were performed using Stata (V.15; StataCorp, College Station, Texas, USA) with a two-sided test with $\alpha=0.05$. Descriptive statistics were obtained in the participants included in this study in comparison with those excluded from this study. Cohen's kappa statistics were calculated to assess the agreement between the FLI and NAFLD score.

MDS, as a measure of adherence to the Mediterranean diet, was evaluated both categorically (quintiles) and continuously scaled as $1 \mathrm{SD}$ unit. The association of MDS with the risk of hepatic steatosis was assessed using multivariable-adjusted Poisson regression models with robust SEs and estimating risk ratios (RRs) and 95\% CIs. Models were adjusted for age, sex, marital status, occupational status, educational level, smoking status, energy intake, total energy expenditure and date of dietary assessment (to adjust for seasonality). We further adjusted for BMI and waist circumference as potential confounders or factors on the causal pathway to assess the possible impact of overall and central adiposity on the association of the Mediterranean diet and hepatic steatosis.

Additionally, we also adjusted for changes in BMI categories between baseline and follow-up; for alcohol consumption (units/week); for clinical variables of metabolic risk (blood pressure $>130 / 85 \mathrm{~mm} \mathrm{Hg}$ (yes/no), triglycerides $>1.7 \mathrm{mmol} / \mathrm{L}$ (yes/no), high-density lipoprotein level
$<1.29 \mathrm{mmol} / \mathrm{L}$ for men and $<1.03 \mathrm{mmol} / \mathrm{L}$ for women (yes/no), and glucose level $\geq 5.6 \mathrm{mmol} / \mathrm{L}($ yes $/ \mathrm{no}))^{38}$; and for family history of diabetes and metabolic syndrome (only for FLI) to examine their influence on the association of interest.

Possible interactions between MDS and age, sex, BMI and alcohol consumption were tested using the Wald test. Several sensitivity analyses were conducted to examine the robustness of the observed findings. First, to assess the role of alcohol consumption (as alcohol is a risk factor for fatty liver accumulation), we excluded the alcohol component from the MDS, while adjusting for alcohol consumption as a covariate. We took the same approaches for the other MDS components to assess the impact of each component on the observed associations. Second, we conducted separate analyses after excluding participants with $\mathrm{BMI} \geq 30 \mathrm{~kg} / \mathrm{m}^{2}$; implausible energy intake $(<500$ or $>3500 \mathrm{kcal} /$ day in women and $<800$ or $>4000 \mathrm{kcal} /$ day in men); excessive alcohol consumption; prevalent diabetes (defined as glycated haemoglobin $\geq 48 \mathrm{mmol} / \mathrm{mol}$, or fasting plasma glucose $\geq 7.0 \mathrm{mmol} / \mathrm{L}$, or use of hypoglycaemic drugs or insulin); or probable secondary causes of hepatic steatosis such as hepatitis B or C, HIV, hepatotoxic or autoimmune disease medications. We evaluated the robustness of the results to an alternative definition of prevalent hepatic steatosis. While we excluded participants with prevalent hepatic steatosis using the specified cut-offs of FLI or NAFLD score in the primary analysis, we used each of the two indices separately in sensitivity analyses, whereby we evaluated 2652 adults in longitudinal analysis based on FLI; and 2568 adults, based on NAFLD. Finally, we used more restrictive cut-points and excluded participants with NAFLD score $\geq-0.640$ or with FLI $>30$.

In a post-hoc analysis, due to inconsistency of the associations observed for FLI and NAFLD score, we also calculated the Hepatic Steatosis Index (HSI $)^{40}$ based on the ratio of AST/ALT, BMI, presence of type 2 diabetes and sex:

$\mathrm{HSI}=8 \times \mathrm{AST} / \mathrm{ALT}+\mathrm{BMI}+2$ (presence of diabetes) +2 (if women).

Presence of hepatic steatosis was defined by an HSI $>36$. After excluding participants with HSI $>36$ at baseline $(\mathrm{n}=2674)$, we evaluated 2351 adults.

In a post-hoc analysis pertaining to sensitivity of the results to model covariates and for better understanding of potential mechanisms, longitudinal associations of MDS with follow-up measures of log-transformed GGT, ALT and AST levels, and with changes in BMI and waist circumference from baseline to follow-up were examined using multivariable-adjusted linear regression. These results were expressed as $\beta$ coefficient (95\% CIs) for changes in each measure per 1 SD difference in MDS.

\section{RESULTS}

\section{Participant characteristics}

Of the initial 5064 participants, 2776 (54.8\%) were excluded, leaving 2288 participants $(65.4 \%$ women; 
$55.8 \pm 10.0$ years) for analysis. Participants included were more likely to be women, show higher sociodemographic characteristics and lower BMI, waist circumference, liver enzymes or prevalence of metabolic syndrome in comparison with excluded individuals (online supplemental table S1). Being in the highest quintile of MDS was higher among women compared with men, positively correlated with sociodemographic characteristics, and negatively correlated with being current smokers, heavy alcohol drinkers, and with BMI, waist circumference, GGT and TG (table 1).

\section{Adherence to the Mediterranean diet and risk of hepatic steatosis}

After a mean 5.3 (SD: 0.5) years of follow-up, there were $153(6.7 \%)$ and $208(9.1 \%)$ participants with hepatic steatosis based on FLI and NAFLD score, respectively (online supplemental table S2). Case identification by FLI and NAFLD score was modestly concordant (kappa $=0.60)$.

Multivariable-adjusted analysis showed an inverse association between MDS quintiles and risk of hepatic steatosis based on FLI $\left(p_{\text {trend }}<0.006\right)$ with RR $(95 \%$ CI) comparing the top to the bottom category of $0.50(0.28$ to 0.91 ). The inverse associations across quintiles of MDS weakened after adjustment for BMI $\left(\mathrm{p}_{\text {trend }}=0.031\right)$ or both BMI and waist circumference ( $\mathrm{p}_{\text {trend }}=0.034$ ) (table 2): RR $(95 \% \mathrm{CI})=0.61$ (0.34 to 1.09$)$ and 0.60 (0.34 to 1.08$)$, respectively. In analyses using MDS as a continuous variable, the inverse association with risk of hepatic steatosis based on FLI (0.84 (0.73 to 0.96) per 1 SD of MDS) remained unchanged but getting imprecise after adjustment for BMI (0.85 (0.73 to 0.99)) and adjustment for both BMI and waist circumference $(0.85$ (0.71 to 1.02$)$ ). In sensitivity analysis, the magnitude of the inverse associations changed a little after further adjustment for alcohol consumption, presence of metabolic syndrome, changes in BMI categories or clinical variables (medication use or prevalent diseases), while adjustment for BMI and clinical variables increased SEs (online supplemental table S3).

Conversely, there was no association between MDS and the risk of hepatic steatosis defined by NAFLD score criteria, with RRs (95\% CIs) ranging from 0.93 (0.82 to 1.05 ) to 1.00 (0.86 to 1.17 ) over different regression models (table 2 and online supplemental table S3).

\section{Interaction and sensitivity analyses}

No significant interactions were found between MDS and age, sex, BMI or alcohol consumption on risk of hepatic steatosis $\left(\mathrm{p}_{\text {interaction }}>0.05\right.$; results not shown $)$. The contribution of each component of the MDS on risk of hepatic steatosis was assessed by sequential subtraction of components from the score (figure 1). Excluding the components of the MDS did not substantially affect the inverse associations with hepatic steatosis based on FLI; the magnitude of the associations remained reasonably stable, but it became weaker $(\mathrm{p}>0.05)$ after excluding fruits, cereals, dairy products, red or processed meat, or alcohol.

In sensitivity analyses, when excluding the alcohol component from the MDS but adjusting for alcohol consumption as a covariate, the inverse associations between MDS and risk of hepatic steatosis based on FLI became weaker (online supplemental table S4). The primary results were not different when excluding participants with BMI $\geq 30 \mathrm{~kg} / \mathrm{m}^{2}$, excessive alcohol consumption or secondary causes of hepatic steatosis (online supplemental table S4). Excluding participants with implausible energy intakes weakened the associations (online supplemental table S4). The analysis of an alternative definition of prevalent hepatic steatosis, excluding participants with only high FLI score at baseline, did not alter the significant inverse association between MDS and risk of FLI-based hepatic steatosis (online supplemental table S5). In post-hoc analyses, there was an inverse association between MDS quintiles and risk of hepatic steatosis based on HSI ( $\left.\mathrm{p}_{\text {trend }}=0.070\right)$ with RR (95\% CI) comparing the top with the bottom category of 0.70 (0.55 to 0.91 ); a significant inverse association with HSI was observed per 1 SD increase in MDS (0.90 (0.82 to 0.98)) (online supplemental table S5). Effect sizes were of slightly higher magnitude when excluding those with FLI $>30$ or NAFLD score $\geq-0.640$ at baseline, but CIs were wider due to smaller sample size (online supplemental table S6).

For NAFLD score, no significant associations were found in any of the sensitivity analyses (online supplemental tables S4 and S5). The sole exception was when participants with a high NAFLD score at baseline were excluded, where an inverse association between MDS quintiles and risk of hepatic steatosis was present $\left(\mathrm{p}_{\text {trend }}=0.039\right)$, but this association was attenuated to the null after adjustment for BMI (online supplemental table S5).

\section{Longitudinal analyses for adiposity and markers of hepatic function}

In post-hoc exploratory analyses, there were inverse associations of MDS with changes in BMI ( $\beta$ coefficient $(95 \%$ CIs) per 1 SD higher MDS of -0.08 (-0.15 to -0.02$))$ and in waist circumference $(-0.33(-0.61$ to -0.06$))$ (online supplemental table S7). For the markers of hepatic function, MDS showed a trend toward inverse association with GGT levels $\left(\mathrm{p}_{\text {trend }}=0.047\right.$ ) ( $\beta$ coefficient $(95 \%$ CIs) per 1 SD higher MDS of -1.66 (-3.73 to 0.41$)$ ), but not with ALT or AST levels (online supplemental table S8).

\section{DISCUSSION}

In this first population-based European study among adults free from clinically manifest hepatic steatosis to report on the prospective association between adherence to the Mediterranean diet and risk of hepatic steatosis, we found an inverse association between MDS and risk of hepatic steatosis based on FLI criteria. This relationship was attenuated to the null when controlled for general and central adiposity assessed by the BMI and 
Table 1 Baseline characteristics of participants according to quintiles of the Mediterranean diet score, CoLaus Study, Switzerland $(n=2288)$

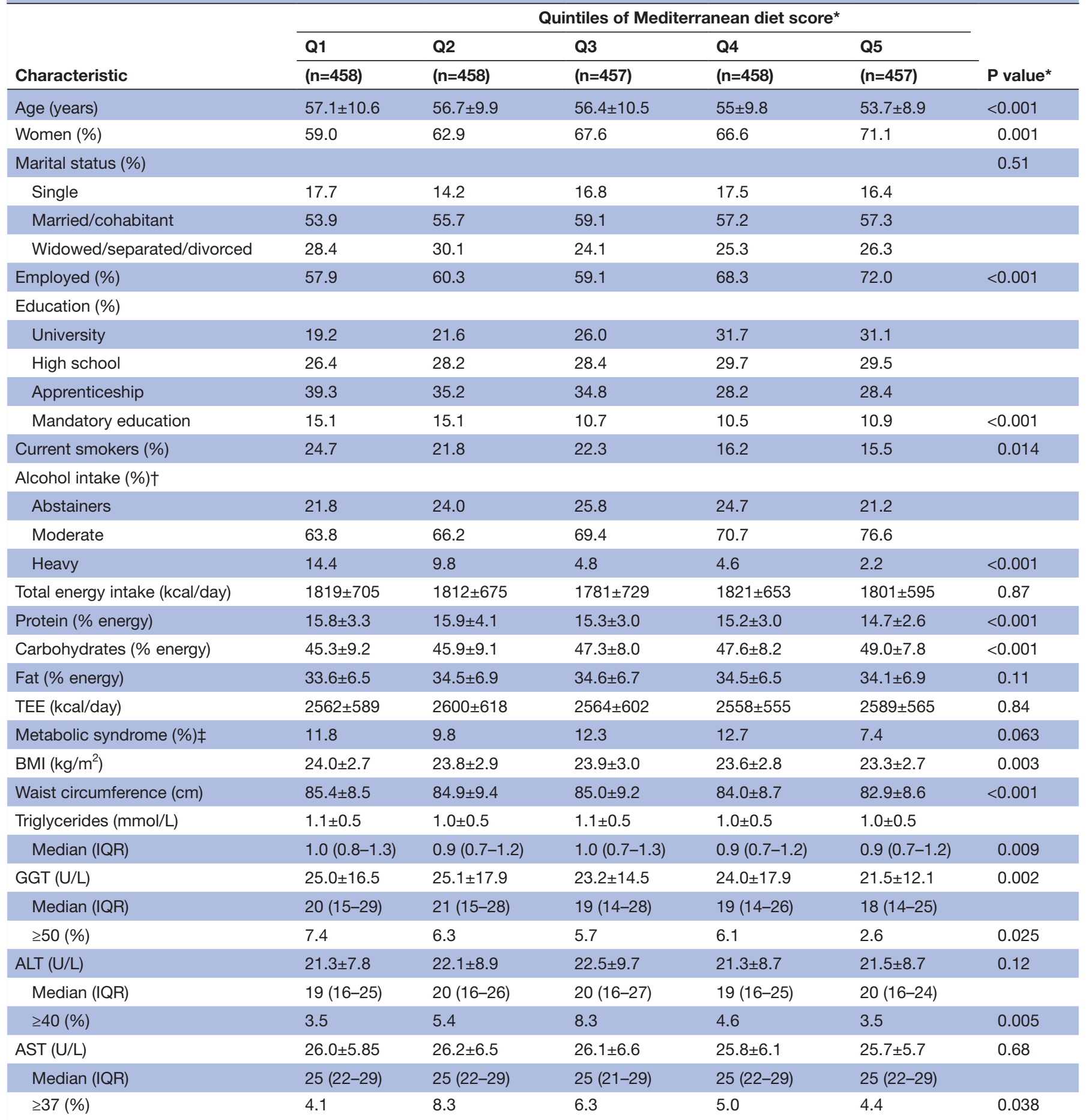

Data are mean \pm SD for continuous variables or percent for categorical variables, unless otherwise stated.

*The population was divided into five groups by quintiles (Q1-Q5) of the Mediterranean diet score. P values were computed by using ANOVA for continuous variables and $\mathrm{X}^{2}$ test for categorical variables.

†Alcohol consumption categorised as 'abstainers' (0 unit/week), 'moderate' (1-21 units/week for men, 1-14 for women) and 'heavy drinkers' (>21 units/week for men, $>14$ for women).

¥Metabolic syndrome defined according to the International Diabetes Federation (waist circumference $\geq 94 \mathrm{~cm}$ in men and $\geq 80 \mathrm{~cm}$ in women plus at least two of the following factors: serum triglycerides $\geq 1.70 \mathrm{mmol} / \mathrm{L}$ or specific treatment for this lipid abnormality; serum high-density lipoprotein cholesterol $<1.03 \mathrm{mmol} / \mathrm{L}$ in men and $<1.29 \mathrm{mmol} / \mathrm{L}$ in women or specific treatment for this lipid abnormality; systolic blood pressure $\geq 130 \mathrm{~mm} \mathrm{Hg}$ or diastolic blood pressure $\geq 85 \mathrm{~mm} \mathrm{Hg}$ or treatment for previously diagnosed hypertension; and fasting plasma glucose $\geq 5.6 \mathrm{mmol} / \mathrm{L}$ or previously diagnosed type 2 diabetes).

ALT, alanine aminotransferase; ANOVA, analysis of variance; AST, aspartate aminotransferase; BMI, body mass index; GGT, gamma-glutamyl transferase; IQR, interquartile range; TEE, total energy expenditure. 


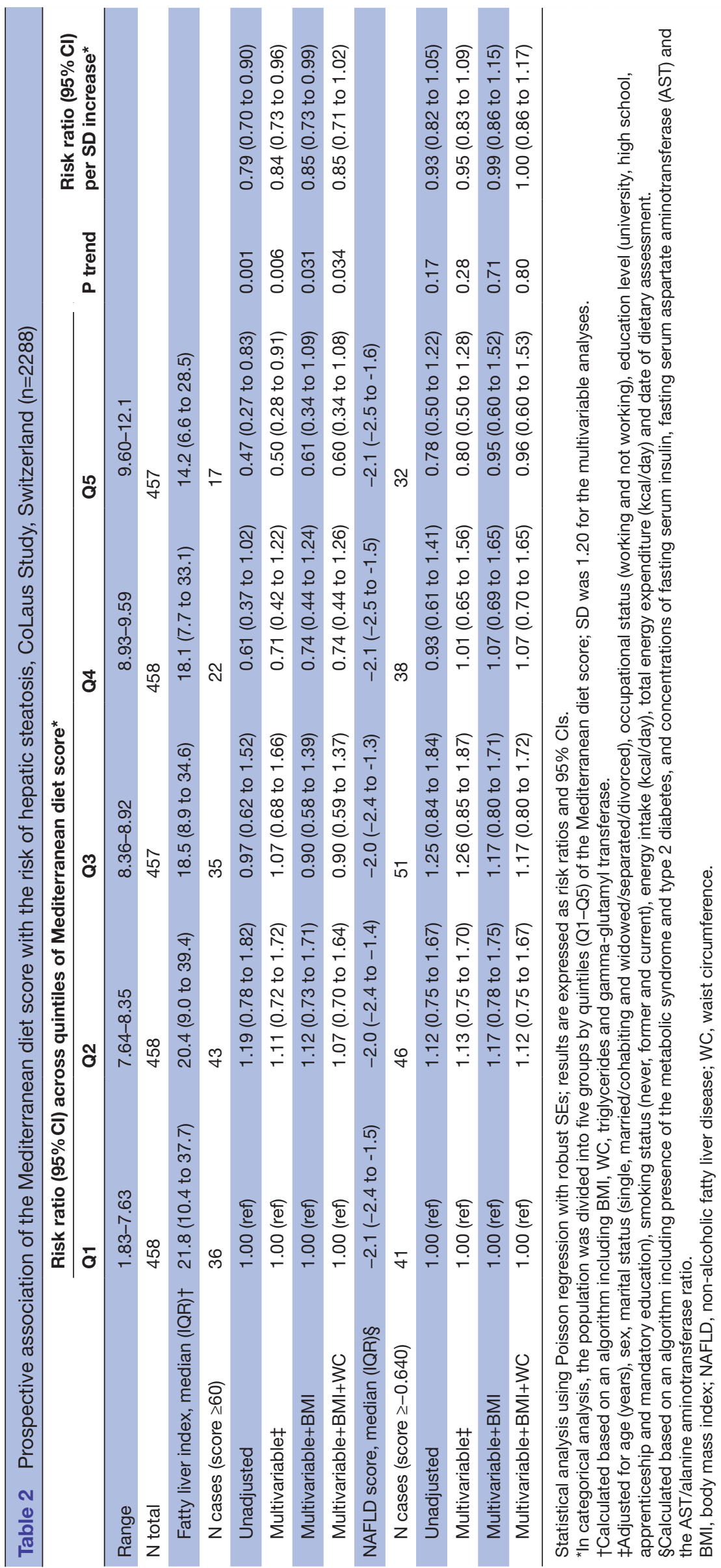


FLI, unadjusted model

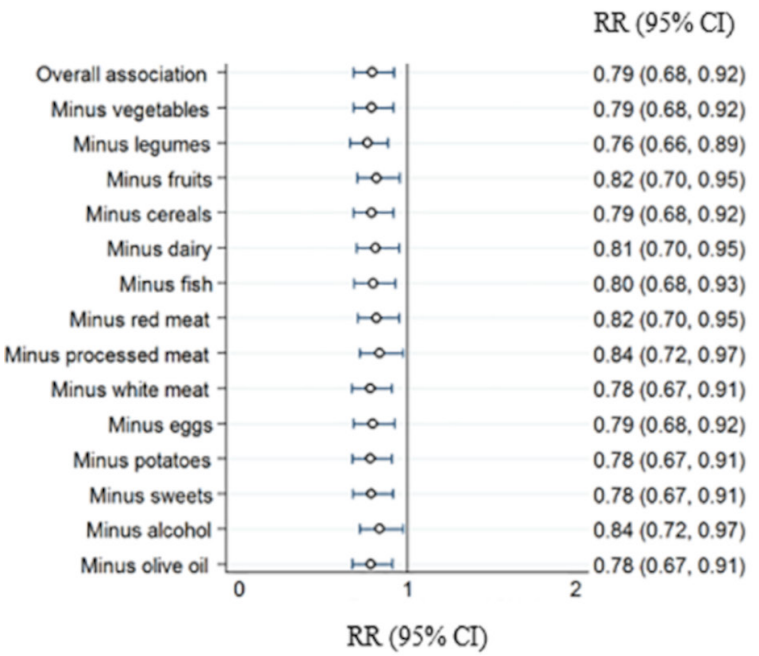

NAFLD-score, unadjusted model

$\mathrm{RR}(95 \% \mathrm{CI})$

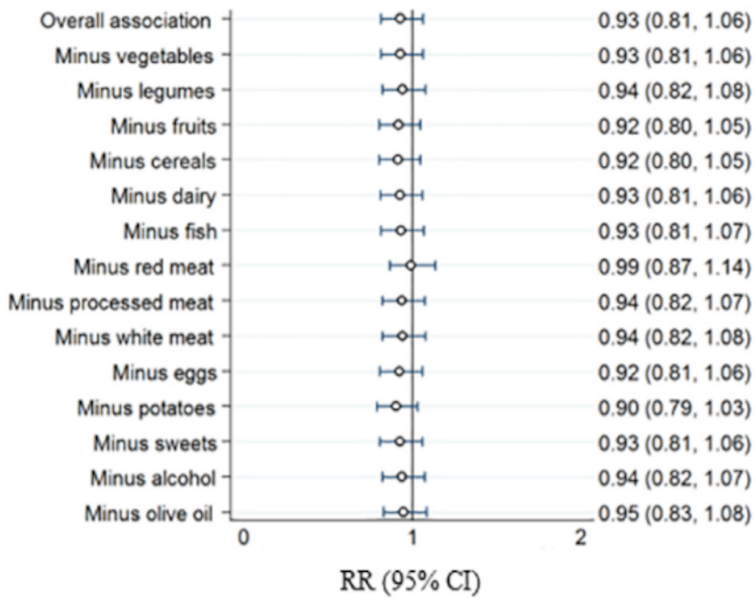

FLI, multivariable adjusted model*

$R R(95 \% \mathrm{CI})$

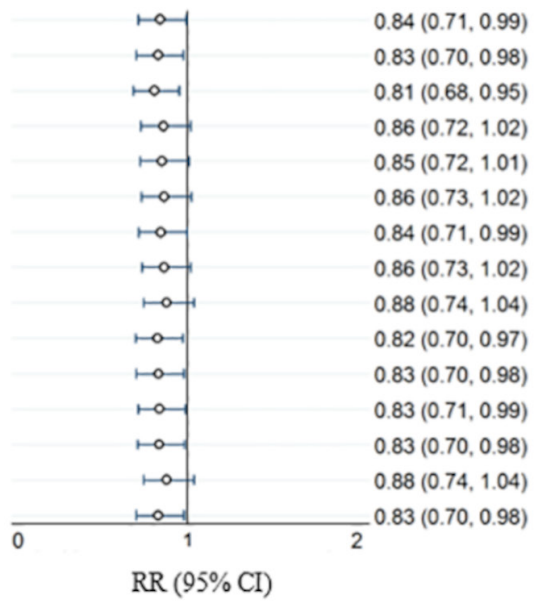

NAFLD-score, multivariable adjusted model*

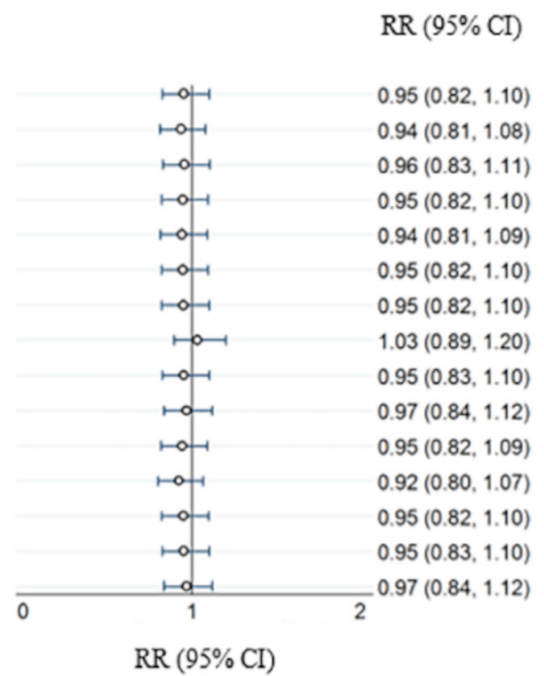

Figure 1 Prospective association of the Mediterranean diet score (MDS) with the risk of hepatic steatosis, CoLaus Study, Switzerland $(n=2288)$ : sensitivity analysis to examine influence of each component of Mediterranean diet. Statistical analysis using Poisson regression with robust SEs; results are expressed as risk ratios (RRs) and 95\% Cls. RRs and 95\% Cls were estimated per 1 SD of MDS (overall association) or of each MDS computed after excluding one component. *Adjusted for age (years), sex, marital status (single, married/cohabiting and widowed/separated/divorced), occupational status (working and not working), education level (university, high school, apprenticeship and mandatory education), smoking status (never, former and current), energy intake (kcal/day), total energy expenditure (kcal/day) and date of dietary assessment. FLI, Fatty Liver Index; NAFLD, non-alcoholic fatty liver disease.

waist circumference. In contrast, there was no association between adherence to the Mediterranean diet and risk of hepatic steatosis based on NAFLD score criteria.

\section{Current findings in context of other evidence}

Our finding based on FLI is consistent with the only published prospective study relating the Mediterranean diet to hepatic steatosis, where each SD increase in MDS was estimated to decrease the odds for incident hepatic steatosis by $26 \%$ (95\% CI $10 \%$ to $39 \%$ ). ${ }^{28}$ By contrast, the point estimate of the effect size in our study was smaller (16\% (95\% CI $4 \%$ to $27 \%)$ ). A possible explanation partly lies in methodological differences. We used biochemical and anthropometric markers to estimate hepatic steatosis in the current study, while the previous study used CT assessment.

No association was found between adherence to the Mediterranean diet and risk of hepatic steatosis based on the NAFLD score. For possible explanations based on the differences in their components, the FLI includes GGT, while NAFLD score includes AST and AST/ALT ratio; the FLI includes adiposity markers, while the NAFLD score does not; the FLI includes a lipid marker (triglycerides) while NAFLD score includes markers of glycaemic status. Previous studies showed a modest association of GGT and ALT (but not AST) with the prevalence of hepatic steatosis. ${ }^{1}$ Indeed, our analysis showed an inverse 
association between MDS and GGT levels, but not with AST or ALT, and these findings could explain the discrepancy between the two indices. Notably, our sensitivity analyses using different definitions of hepatic steatosis showed an inverse association between MDS quintiles and risk of hepatic steatosis based on NAFLD score after excluding participants with high baseline NAFLD score only. This could be explained by modest concordance between the two measures. Of note, there were no statistically significant associations between MDS and risk of hepatic steatosis based on FLI after excluding participants with FLI $>30$ at baseline, which led to a smaller sample size and consequently a lower statistical power in our study. In a post-hoc analysis, we found an inverse association between adherence to the Mediterranean diet and hepatic steatosis as assessed by HSI, an alternative score to detect hepatic steatosis. Although case identification by HSI and FLI (kappa=0.27) or NAFLD score (kappa $=0.18$ ) was weakly concordant, our finding based on FLI is consistent with HSI. This could be explained potentially by BMI as one of the components of both FLI and HSI, highlighting the importance of obesity for incident hepatic steatosis. ${ }^{41}$

The inverse association between MDS and risk of hepatic steatosis defined by FLI remained significant after adjusting for BMI only, but became imprecise and not significant after adjusting for both BMI and waist circumference or for changes in BMI over the follow-up period. These results suggest the collinearity between the central adiposity and hepatic steatosis as the central adiposity may partly reflect hepatic steatosis. This biologically plausible finding is in agreement with previous cross-sectional findings for MDS and prevalent hepatic steatosis in CoLaus Study and the British Fenland Study we reported, ${ }^{27}$ and a study in Hong Kong. ${ }^{42}$ Our finding that the MDS was negatively associated with an increase in BMI after 5.3 years of follow-up is consistent with the findings from the Framingham Heart Study which observed the same trend over 6-year follow-up, ${ }^{28}$ and from Nurses' Health Study and Health Professionals' Follow-up Study evaluating 20-year longitudinal data with repeated selfreported measures of diet and adiposity measures. ${ }^{43}$

Sequential subtraction of different components of the MDS showed that fruits, cereals, dairy products, red or processed meat, or alcohol partially accounted for the observed association. These findings agree with previous studies ${ }^{26} 284445$ including the Framingham Heart Study suggesting benefits of low consumption of red meat and high consumption of fruits or whole grains ${ }^{28}$; and a cross-sectional analysis from the PREDIMED Study suggesting the benefit of low consumption of red meat. ${ }^{26}$ Moreover, the Mediterranean diet is characterised by a moderate-to-high consumption of whole grains, which has been inversely associated with the likelihood of having NAFLD. ${ }^{46}$

\section{Possible mechanisms and implications}

Hepatic steatosis is associated with a number of metabolic risk factors including insulin resistance, type 2 diabetes, dyslipidaemia, metabolic syndrome and oxidative stress. ${ }^{47}$ Mediterranean diet-associated phenolic compounds (phenolic acids and polyphenols) found in fruits and vegetables and high levels of monounsaturated fatty acids of olive oil have been shown to inhibit de novo lipogenesis, improve peripheral insulin sensitivity, and reduced cardiovascular risk mainly due to their antioxidant, anti-inflammatory and anti-fibrotic effects. ${ }^{18}$ 48-51 Moreover, different components of the Mediterranean diet, including omega-3 polyunsaturated fatty acids (PUFA), fibre and antioxidant rich-foods are inversely associated with hepatic steatosis. ${ }^{52}{ }^{53}$ One meta-analysis of interventional studies reported that omega-3 PUFA were negatively associated with hepatic steatosis. ${ }^{54}$ The Mediterranean diet is also low in saturated fat, which has been demonstrated to increase hepatic triglycerides content and hepatic insulin resistance. ${ }^{55}{ }^{56}$ Finally, the high-fibre content of the Mediterranean diet has been associated with reduced hepatic fat. ${ }^{1852}$

Hepatic steatosis is associated with cardiometabolic diseases and substantially impacts public health. ${ }^{13} 1516$ Thus, our finding of an inverse association between adherence to the Mediterranean diet and risk of hepatic steatosis would support the importance of dietary advice for the prevention of hepatic steatosis as well as its treatment. However, future work should confirm whether or not the clinical importance of the Mediterranean diet for the prevention of hepatic steatosis is independent of obesity or central adiposity.

\section{Strengths and limitations}

To our knowledge, this is the first European prospective study assessing the association between the Mediterranean diet and risk of hepatic steatosis. The study had the benefit of a relatively large sample size and an average of 5.3 years of follow-up, and applied a definition of the Mediterranean diet that has been shown to be valid in a non-Mediterranean population. ${ }^{33}$

Several limitations of this study merit consideration. Measurement error and recall bias are inevitable when using self-reported dietary instruments, limiting the ability to precisely measure adherence to the Mediterranean diet, although adjustment for energy intake may have reduced the magnitude of measurement error. ${ }^{57}$ We used diet data measured only at baseline but recognised that intraindividual variation over time might be present which would be expected to weaken the observed associations, and hence our findings may be biased towards the null. However, in CoLaus, average change in estimated total energy intake from first to second follow-up was $51 \mathrm{kcal} /$ day and changes for each macronutrient (expressed as \% of total energy intake) were about $1 \%$ (data not shown). Thus, dietary intake in CoLaus was relatively stable, suggesting that the lack of availability of repeat dietary measures is unlikely to alter our findings substantially.

Our ascertainment of hepatic steatosis was based on two indices, but not on liver biopsy or direct imaging assessment; hence, information bias (misdiagnosis) cannot be 
ruled out. Although liver biopsy is the gold standard for diagnosing hepatic steatosis, its use in apparently healthy participants would be unethical and unfeasible in a large population-based study. Further, previous studies have shown that FLI and NAFLD score can accurately identify hepatic steatosis with good sensitivity and specificity, and these scores have been validated for use in large epidemiological studies. ${ }^{36} 375859$ But, although both FLI and NAFLD score can indicate hepatic steatosis, their precision is variable. ${ }^{60}$ Moreover, presence of steatohepatitis or advanced liver fibrosis could influence the relationship of FLI or NAFLD score with hepatic steatosis. ${ }^{61}$ Of note, our study was not designed to assess the role of adherence to the Mediterranean diet in hepatic fibrosis.

Although we adjusted for many relevant confounders and performed a series of sensitivity analyses, we cannot rule out residual confounding due to unmeasured variables or covariates measured with error. On the other hand, our adjustment for BMI and waist circumference as markers of general and central adiposity may potentially be an overadjustment if adiposity is on the causal pathway between dietary adherence and hepatic steatosis. However, since FLI may approximate hepatic steatosis with a degree of imprecision, adjusting for adiposity in these analyses may not represent adjusting the association between diet and steatosis directly, but through an HSI that already includes adiposity measures in its definition. Nevertheless, our analytical approach is comprehensive, showing the results for crude analyses, followed by multivariable adjustment without and with further adjustment for adiposity markers. Future research with repeat measurements should further investigate this issue. Generalisability of our findings is limited because included participants seemed to be healthier than those excluded, and our findings were obtained in a single European population. Still, they confirm the findings from a previous prospective study conducted in the USA ${ }^{28}$ and might serve as a reference for other studies.

\section{CONCLUSION}

Adherence to the Mediterranean diet was inversely associated with risk of hepatic steatosis based on the FLI, and the association was independent of several known risk factors. Conversely, the association was not observed when using different criteria specifying the NAFLD score. These findings support recommendations on following the Mediterranean diet for hepatic steatosis prevention in addition to the existing evidence for its benefit for CVD prevention. Nonetheless, the findings also highlight the need for further research with more accurate measures of hepatic steatosis to replicate these findings in different populations and settings.

\section{Author affiliations}

${ }^{1}$ Faculty of Medicine and Health, The University of Sydney School of Public Health, Sydney, New South Wales, Australia

${ }^{2}$ ARC Centre for Excellence in Population Ageing Research (CEPAR), University of Sydney, Sydney, New South Wales, Australia
${ }^{3}$ Department of Internal Medicine, Internal Medicine, University Hospital of Lausanne (CHUV), Lausanne, Switzerland

${ }^{4}$ Medical Research Council Epidemiology Unit, University of Cambridge, School of Clinical Medicine, Cambridge, UK

\section{Twitter Saman Khalatbari-Soltani @Saamaankh}

Acknowledgements The authors are grateful to all the participants and staff of CoLaus Study.

Contributors SK-S, Fl and NGF designed the study question, and had full access to all the data in the study and took responsibility for the integrity and accuracy of the data. SK-S performed the statistical analyses and wrote the first draft with supervision from FI, PM-V and NGF. All authors contributed to interpretation of data, revised the article critically for important intellectual content and approved the final version of the manuscript.

Funding The CoLaus Study was and is supported by research grants from GlaxoSmithKline, the Faculty of Biology and Medicine of Lausanne, and the Swiss National Science Foundation (grants 33CSC0-122661, 33CS30-139468 and 33CS30-148401). SK-S was supported by the Swiss National Science Foundation (Doc.Mobility number P1LAP3-171805). NGF and Fl acknowledge core MRC support (MC_UU_12015/5), and NGF acknowledges NIHR Biomedical Research Centre Cambridge: Nutrition, Diet and Lifestyle Research Theme (IS-BRC-1215-20014).

Competing interests None declared.

\section{Patient consent for publication Not required.}

Ethics approval The Institutional Ethics Committee of the University of Lausanne, which afterwards became the Ethics Commission of Canton Vaud (www.cer-vd.ch) approved the baseline CoLaus Study (reference 16/03); the approval was renewed for the first (reference 33/09) and the second (reference 26/14) follow-ups. The CoLaus Study was performed in agreement with the Helsinki Declaration and its former amendments, and all participants provided their written informed consent before entering the study.

Provenance and peer review Not commissioned; externally peer reviewed.

Data availability statement Data are available upon reasonable request. Non-identifiable individual-level data are available for researchers who seek to answer questions related to health and disease in the context of research projects who meet the criteria for data sharing by research committees. Please follow the instructions at https://www.colaus-psycolaus.ch/ for information on how to submit an application for gaining access to CoLaus data.

Supplemental material This content has been supplied by the author(s). It has not been vetted by BMJ Publishing Group Limited (BMJ) and may not have been peer-reviewed. Any opinions or recommendations discussed are solely those of the author(s) and are not endorsed by BMJ. BMJ disclaims all liability and responsibility arising from any reliance placed on the content. Where the content includes any translated material, BMJ does not warrant the accuracy and reliability of the translations (including but not limited to local regulations, clinical guidelines, terminology, drug names and drug dosages), and is not responsible for any error and/or omissions arising from translation and adaptation or otherwise.

Open access This is an open access article distributed in accordance with the Creative Commons Attribution 4.0 Unported (CC BY 4.0) license, which permits others to copy, redistribute, remix, transform and build upon this work for any purpose, provided the original work is properly cited, a link to the licence is given, and indication of whether changes were made. See: https://creativecommons.org/ licenses/by/4.0/.

\section{ORCID iDs}

Saman Khalatbari-Soltani http://orcid.org/0000-0001-8437-1906

Pedro Marques-Vidal http://orcid.org/0000-0002-4548-8500

Fumiaki Imamura http://orcid.org/0000-0002-6841-8396

Nita G. Forouhi http://orcid.org/0000-0002-5041-248X

\section{REFERENCES}

1 Sattar N, Forrest E, Preiss D. Non-alcoholic fatty liver disease. BMJ 2014;349:g4596.

2 Angulo P. Nonalcoholic fatty liver disease. N Engl J Med 2002;346:1221-31.

3 Williamson RM, Price JF, Glancy S, et al. Prevalence of and risk factors for hepatic steatosis and nonalcoholic fatty liver disease in 
people with type 2 diabetes: the Edinburgh type 2 diabetes study. Diabetes Care 2011;34:1139-44

4 Bellentani S, Scaglioni F, Marino M, et al. Epidemiology of nonalcoholic fatty liver disease. Dig Dis 2010;28:155-61.

5 Angulo P, Keach JC, Batts KP, et al. Independent predictors of liver fibrosis in patients with nonalcoholic steatohepatitis. Hepatology 1999;30:1356-62.

6 Marchesini G, Petta S, Dalle Grave R. Diet, weight loss, and liver health in nonalcoholic fatty liver disease: pathophysiology, evidence, and practice. Hepatology 2016;63:2032-43.

7 European Association for the Study of the Liver (EASL), European Association for the Study of Diabetes (EASD), European Association for the Study of Obesity (EASO). EASL-EASD-EASO clinical practice guidelines for the management of non-alcoholic fatty liver disease. $J$ Hepatol 2016;64:1388-402.

8 Chalasani N, Younossi Z, Lavine JE, et al. The diagnosis and management of non-alcoholic fatty liver disease: practice guideline by the American association for the study of liver diseases, American College of gastroenterology, and the American gastroenterological association. Hepatology 2012;55:2005-23.

9 Sawangjit R, Chongmelaxme B, Phisalprapa P, et al. Comparative efficacy of interventions on nonalcoholic fatty liver disease (NAFLD): a PRISMA-compliant systematic review and network meta-analysis. Medicine 2016;95:e4529.

10 Plauth M, Bernal W, Dasarathy S, et al. ESPEN guideline on clinical nutrition in liver disease. Clin Nutr 2019;38:485-521.

11 Abenavoli L, Di Renzo L, Boccuto L, et al. Health benefits of Mediterranean diet in nonalcoholic fatty liver disease. Expert Rev Gastroenterol Hepatol 2018;12:873-81.

12 Asbaghi O, Choghakhori R, Ashtary-Larky D, et al. Effects of the Mediterranean diet on cardiovascular risk factors in non-alcoholic fatty liver disease patients: a systematic review and meta-analysis. Clin Nutr ESPEN 2020;37:148-56.

13 Estruch R, Ros E, Salas-Salvadó J, et al. Primary prevention of cardiovascular disease with a Mediterranean diet supplemented with extra-virgin olive oil or nuts. N Engl J Med 2018;378:e34.

14 Sofi F, Cesari F, Abbate R, et al. Adherence to Mediterranean diet and health status: meta-analysis. BMJ 2008;337:a1344.

15 Salas-Salvadó J, Bulló M, Babio N, et al. Reduction in the incidence of type 2 diabetes with the Mediterranean diet: results of the PREDIMED-Reus nutrition intervention randomized trial. Diabetes Care 2011;34:14-19.

16 Esposito K, Kastorini C-M, Panagiotakos DB, et al. Mediterranean diet and metabolic syndrome: an updated systematic review. Rev Endocr Metab Disord 2013;14:255-63.

17 Ryan MC, Itsiopoulos C, Thodis T, et al. The Mediterranean diet improves hepatic steatosis and insulin sensitivity in individuals with non-alcoholic fatty liver disease. J Hepatol 2013;59:138-43.

18 Properzi C, O'Sullivan TA, Sherriff JL, et al. Ad libitum Mediterranean and low-fat diets both significantly reduce hepatic steatosis: a randomized controlled trial. Hepatology 2018;68:1741-54.

19 Misciagna G, Del Pilar Díaz M, Caramia DV, et al. Effect of a low glycemic index Mediterranean diet on non-alcoholic fatty liver disease. A randomized controlled clinici trial. J Nutr Health Aging 2017;21:404-12.

20 Trovato FM, Catalano D, Martines GF, et al. Mediterranean diet and non-alcoholic fatty liver disease: the need of extended and comprehensive interventions. Clin Nutr 2015;34:86-8.

21 Gelli C, Tarocchi M, Abenavoli L, et al. Effect of a counselingsupported treatment with the Mediterranean diet and physica activity on the severity of the non-alcoholic fatty liver disease. World $J$ Gastroenterol 2017;23:3150-62.

22 Baratta F, Pastori D, Polimeni L, et al. Adherence to Mediterranean diet and non-alcoholic fatty liver disease: impact on metabolic profile. Nutr Metab Cardiovasc Dis 2017;27:e8

23 Abenavoli L, Greco M, Nazionale I, et al. Effects of Mediterranean diet supplemented with silybin-vitamin E-phospholipid complex in overweight patients with non-alcoholic fatty liver disease. Expert Rev Gastroenterol Hepatol 2015;9:519-27.

24 Fraser A, Abel R, Lawlor DA, et al. A modified Mediterranean diet is associated with the greatest reduction in alanine aminotransferase levels in obese type 2 diabetes patients: results of a quasirandomised controlled trial. Diabetologia 2008;51:1616-22.

25 Kontogianni MD, Tileli N, Margariti A, et al. Adherence to the Mediterranean diet is associated with the severity of non-alcoholic fatty liver disease. Clin Nutr 2014;33:678-83.

26 Cantero I, Abete I, Babio N, et al. Dietary inflammatory index and liver status in subjects with different adiposity levels within the PREDIMED trial. Clin Nutr 2018;37:1736-43.

27 Khalatbari-Soltani S, Imamura F, Brage S, et al. The association between adherence to the Mediterranean diet and hepatic steatosis: cross-sectional analysis of two independent studies, the UK Fenland study and the Swiss CoLaus study. BMC Med 2019;17:19.

$28 \mathrm{Ma} \mathrm{J}$, Hennein R, Liu C, et al. Improved diet quality associates with reduction in liver fat, particularly in individuals with high genetic risk scores for nonalcoholic fatty liver disease. Gastroenterology 2018;155:107-17.

29 Firmann M, Mayor V, Vidal PM, et al. The CoLaus study: a population-based study to investigate the epidemiology and genetic determinants of cardiovascular risk factors and metabolic syndrome. BMC Cardiovasc Disord 2008;8:6.

30 Morabia A, Bernstein M, Kumanyika S, et al. Développement et validation d'un questionnaire alimentaire semi-quantitatif partir d'une enquête de population. Soz Praventivmed 1994;39:345-69.

31 Bernstein L, Huot IMA, Bernstein L, et al. Amélioration des performances d'un questionnaire alimentaire semi-quantitatif comparé un rappel des 24 heures. Sante Publique 1995;7:403-13.

32 Bach-Faig A, Berry EM, Lairon D, et al. Mediterranean diet pyramid today. Science and cultural updates. Public Health Nutr 2011;14:2274-84.

33 Tong TYN, Wareham NJ, Khaw K-T, et al. Prospective association of the Mediterranean diet with cardiovascular disease incidence and mortality and its population impact in a non-Mediterranean population: the EPIC-Norfolk study. BMC Med 2016;14:135.

34 Willett WC, Howe GR, Kushi LH. Adjustment for total energy intake in epidemiologic studies. Am J Clin Nutr 1997;65:1220S-8. discussion 1229S-1231S.

35 Bedogni G, Bellentani S, Miglioli L, et al. The fatty liver index: a simple and accurate predictor of hepatic steatosis in the general population. BMC Gastroenterol 2006;6:33.

36 Kotronen A, Peltonen M, Hakkarainen A, et al. Prediction of nonalcoholic fatty liver disease and liver fat using metabolic and genetic factors. Gastroenterology 2009;137:865-72.

37 Ruhl CE, Everhart JE. Fatty liver indices in the multiethnic United States National health and nutrition examination survey. Aliment Pharmacol Ther 2015;41:65-76.

38 Alberti K, Zimmet P, Shaw J. Metabolic syndrome--a new world-wide definition. A consensus statement from the international diabetes federation. Diabet Med 2006;23:469-80.

39 Bernstein M, Sloutskis D, Kumanyika S, et al. Data-based approach for developing a physical activity frequency questionnaire. Am J Epidemiol 1998;147:147-54.

40 Lee J-H, Kim D, Kim HJ, et al. Hepatic steatosis index: a simple screening tool reflecting nonalcoholic fatty liver disease. Dig Liver Dis 2010;42:503-8.

41 Stefan N, Häring $\mathrm{H}-U$, Cusi K. Non-alcoholic fatty liver disease: causes, diagnosis, cardiometabolic consequences, and treatment strategies. Lancet Diabetes Endocrinol 2019;7:313-24.

42 Chan R, Wong VW-S, Chu WC-W, et al. Diet-Quality scores and prevalence of nonalcoholic fatty liver disease: a population study using Proton-Magnetic resonance spectroscopy. PLoS One 2015;10:e0139310-4.

43 Fung $T$, Pan A, Hou T, et al. Long-Term change in diet quality is associated with body weight change in men and women. $J$ Nutr 2015;145:1850-6.

44 Freedman ND, Cross AJ, McGlynn KA, et al. Association of meat and fat intake with liver disease and hepatocellular carcinoma in the NIHAARP cohort. J Natl Cancer Inst 2010;102:1354-65.

45 Georgoulis M, Kontogianni MD, Yiannakouris N. Mediterranean diet and diabetes: prevention and treatment. Nutrients 2014;6:1406-23.

46 Georgoulis M, Kontogianni MD, Tileli N, et al. The impact of cereal grain consumption on the development and severity of non-alcoholic fatty liver disease. Eur J Nutr 2014:53:1727-35.

47 Abenavoli L, Milic N, Di Renzo L, et al. Metabolic aspects of adult patients with nonalcoholic fatty liver disease. World J Gastroenterol 2016;22:7006-16.

48 Salomone F, Godos J, Zelber-Sagi S. Natural antioxidants for non-alcoholic fatty liver disease: molecular targets and clinical perspectives. Liver Int 2016;36:5-20.

49 Abenavoli L, Milic N, Luzza F, et al. Polyphenols treatment in patients with nonalcoholic fatty liver disease. J Trans/ Intern Med 2017;5:144-7.

50 Yang J, Fernández-Galilea M, Martínez-Fernández L, et al. Oxidative stress and non-alcoholic fatty liver disease: effects of omega-3 fatty acid supplementation. Nutrients 2019;11 doi:10.3390/nu11040872

51 Salomone F, Ivancovsky-Wajcman D, Fliss-Isakov N, et al. Higher phenolic acid intake independently associates with lower prevalence of insulin resistance and non-alcoholic fatty liver disease. JHEP Rep 2020;2:100069.

52 Zelber-Sagi S, Salomone F, Mlynarsky L. The Mediterranean dietary pattern as the diet of choice for non-alcoholic fatty liver disease: evidence and plausible mechanisms. Liver Int 2017;37:936-49. 
53 Mahady SE, George J. Exercise and diet in the management of nonalcoholic fatty liver disease. Metabolism 2016;65:1172-82.

54 Parker HM, Johnson NA, Burdon CA, et al. Omega-3 supplementation and non-alcoholic fatty liver disease: a systematic review and meta-analysis. J Hepatol 2012;56:944-51.

55 Hernández Elisa Álvarez, Kahl S, Seelig A, et al. Acute dietary fat intake initiates alterations in energy metabolism and insulin resistance. J Clin Invest 2017;127:695-708 http://ovidsp.ovid.com/ athens/ovidweb.cgi?T=JS\&CSC $=$ Y\&NEWS=N\&PAGE=fulltext\&D= emex\&AN=614623041

56 Bjermo H, Iggman D, Kullberg J, et al. Effects of n-6 PUFAs compared with SFAs on liver fat, lipoproteins, and inflammation in abdominal obesity: a randomized controlled trial. Am J Clin Nutr 2012;95:1003-12.

57 Hu FB, Stampfer MJ, Rimm E, et al. Dietary fat and coronary heart disease: a comparison of approaches for adjusting for total energy intake and modeling repeated dietary measurements. $A m \mathrm{~J}$ Epidemiol 1999;149:531-40.

58 Koehler EM, Schouten JNL, Hansen BE, et al. External validation of the fatty liver index for identifying nonalcoholic fatty liver disease in a population-based study. Clin Gastroenterol Hepatol 2013:11:1201-4.

59 Machado MV, Cortez-Pinto H. Non-invasive diagnosis of nonalcoholic fatty liver disease. A critical appraisal. $J$ Hepatol 2013;58:1007-19.

60 Zelber-Sagi S, Webb M, Assy N, et al. Comparison of fatty liver index with noninvasive methods for steatosis detection and quantification. World J Gastroenterol 2013;19:57-64.

61 Fedchuk L, Nascimbeni F, Pais R, et al. Performance and limitations of steatosis biomarkers in patients with nonalcoholic fatty liver disease. Aliment Pharmacol Ther 2014;40:1209-22. 